\title{
Pantothenate Kinase-Associated Neurodegeneration
}

\author{
Monika B. Hartig ${ }^{1, *}$, Holger Prokisch ${ }^{1,2}$, Thomas Meitinger ${ }^{1,2}$ and Thomas Klopstock ${ }^{3}$ \\ ${ }^{1}$ Institute of Human Genetics, Technische Universität München, Munich, Germany; ${ }^{2}$ Institute of Human Genetics, Helm- \\ holtz Zentrum München, Neuherberg, Germany; ${ }^{3}$ Friedrich-Baur-Institut an der Neurologischen Klinik, Klinikum der \\ Universität München - Innenstadt, Munich, Germany
}

\begin{abstract}
Pantothenate kinase-associated neurodegeneration (PKAN) is a hereditary progressive disorder and the most frequent form of neurodegeneration with brain iron accumulation (NBIA). PKAN patients present with a progressive movement disorder, dysarthria, cognitive impairment and retinitis pigmentosa. In magnetic resonance imaging, PKAN patients exhibit the pathognonomic "eye of the tiger" sign in the globus pallidus which corresponds to iron accumulation and gliosis as shown in neuropathological examinations. The discovery of the disease causing mutations in PANK2 has linked the disorder to coenzyme A (CoA) metabolism. PANK2 is the only one out of four PANK genes encoding an isoform which localizes to mitochondria. At least two other NBIA genes (PLA2G6, C19orf12) encode proteins that share with $P A N K 2$ a mitochondrial localization and all are suggested to play a role in lipid homeostasis.

With no causal therapy available for PKAN until now, only symptomatic treatment is possible. A multi-centre retrospective study with bilateral pallidal deep brain stimulation in patients with NBIA revealed a significant improvement of dystonia. Recently, studies in the PANK Drosophila model "fumble" revealed improvement by the compound pantethine which is hypothesized to feed an alternate CoA biosynthesis pathway. In addition, pilot studies with the iron chelator deferiprone that crosses the blood brain barrier showed a good safety profile and some indication of efficacy. An adequately powered randomized clinical trial will start in 2012.
\end{abstract}

This review summarizes clinical presentation, neuropathology and pathogenesis of PKAN.

Keywords: $P A N K 2$, CoA, iron, neurodegeneration, lipid metabolism, mitochondria, NBIA.

\section{INTRODUCTION}

The heterogeneous group of disorders named neurodegeneration with brain iron accumulation (NBIA) shares the feature of iron deposits in basal ganglia which are detectable by magnetic resonance imaging (MRI). The condition was previously known as Hallervorden-Spatz-Syndrome but the name was abandoned because of unethical activities of these authors in Nazi Germany.

Our understanding of the genetic basis of NBIA has evolved significantly since the identification of $P A N K 2$ in 2001 [1]. Autosomal recessive mutations in PANK2 cause pantothenate kinase-associated neurodegeneration which comprises about $50 \%$ of NBIA cases[2,3]. The prevalence is about one to three in 1,000,000 [4]. Pantothenate kinases are the rate limiting enzymes in the coenzyme A (CoA) biosynthesis pathway and subcellular localization experiments of PANK2 link this pathway to mitochondria [5]. Since the identification of $P A N K 2$, a number of studies have sought to understand the biological role of this enzyme with the aim of advancing treatment of PKAN patients. Even so, only symptomatic treatment is available for PKAN patients so far.

Accumulation of iron in the basal ganglia has provided a pathophysiological overlap between NBIA and common

\footnotetext{
*Address correspondence to this author at the Institute of Human Genetics, Technische Universität München, Munich, Germany; Tel: 0049-89-41406381; Fax: 0049-89-4140-6382;

E-mail: monika.hartig@humangenetik.med.tum.de
}

neurodegenerative diseases like Parkinson (PD) disease [6]. Iron was also identified to be part of amyloid plaques in brain of patients with Alzheimer Disease (AD) [7]. Increased iron levels in brain have been shown to enhance $A \beta$ toxicity in a Drosophila model [8]. Up to now, seven further genes (PLA2G6, FTL, CP, FA2H, ATP13A2, C2orf37, C19orf12) have been linked to NBIA [9-18]. Mutations in two of them (PLA2G6, C19orf12) have been identified in patients with idiopathic Parkinson disease underscoring the commonalities between PD and NBIA at the molecular level $[19,12]$.

In this review we summarize the current understanding of PANK2 dysfunction and clinical manifestations in PKAN.

\section{CLINICAL PHENOTYPE}

Classic PKAN starts in the first decade, usually before age six years. The first symptom is in most instances a gait abnormality. Progressive dystonia is the cardinal symptom of PKAN, and involves primarily the cranial region and the limbs. Dysarthria is another common symptom. Pyramidal tract involvement is also common and may manifest as spasticity, hyperreflexia, and extensor toe signs. Developmental delay beyond motor signs is occasionally encountered, and cognitive impairment correlates with age of onset [20]. Pigmentary retinal degeneration occurs in two-thirds of patients leading to night blindness followed by progressive loss of peripheral visual fields. Optic atrophy, in contrast, and epileptic seizures are rare [21]. In most cases, the disease progresses relentlessly, but the rate of progression does not cor- 
relate in general with age of onset [3]. Most patients with classic PKAN are wheelchair-bound by age 15 years. Premature death is mostly due to secondary complications (most prominently, aspiration pneumonia following dysphagia) but with improved care a number of patients live now into adulthood.

Approximately $25 \%$ of affected individuals have an age of onset later than 10 years (atypical presentation) with a more gradual progression of disease. Speech problems including dysarthria, palilalia and tachylalia may be the presenting feature of patients with atypical PKAN. While later onset correlates with less cognitive impairment [20], psychiatric signs including impulsivity, aggression, depression, emotional lability, and tics are more prevalent [22].

HARP syndrome (hypoprebetalipoproteinemia, acanthocytosis, retinitis pigmentosa, and pallidal degeneration) is now considered part of the PKAN disease spectrum since mutations in the PANK2 gene have been identified in the only two reported HARP families [23].

\section{IMAGING}

Iron deposits in brain cause local magnetic field inhomogeneities with decreased $\mathrm{T} 2$ relaxation time and therefore appear hypointense (dark signal) on T2- and T2*-weighted magnetic resonance imaging (MRI). Importantly, studies of healthy volunteers and Parkinson patients have shown a linear relationship between MRI findings and brain iron concentrations from post mortem studies [24]. While all NBIA forms show the eponymous brain iron, the PKAN MRI is kind of pathognomonic since it shows the "eye of the tiger" sign (Fig. 1). The resemblance is due to a central region of hyperintensity (gliosis) in the center of the hypointense iron accumulation in the globus pallidus [2, 3, 25]. Until now, almost all individuals with $P A N K 2$ mutations have the "eye of the tiger" sign, and almost all individuals with the sign carry PANK2 mutations [25-27]. The MRI changes occur early in the course of the disease and have already been used to predict PKAN in presymptomatic sibs of affected individuals [28].

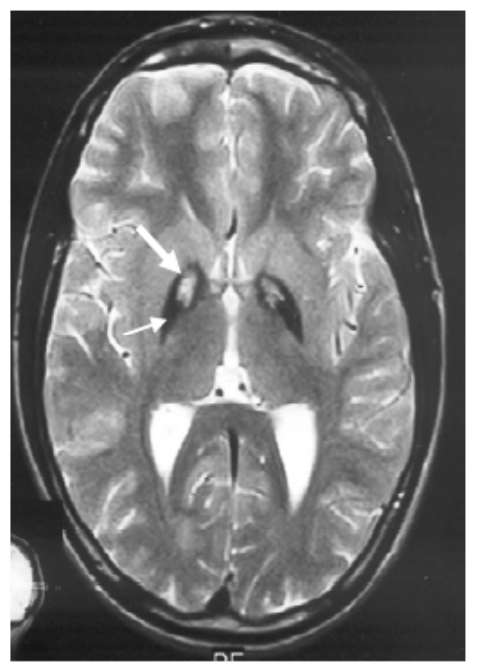

Fig. (1). Axial T2-weighted magnetic resonance images of a PKAN patient. Hypointensity is marked by the thin arrow, the high signal intensity foci in the anteromedial region by the thick arrow (image were kindly contributed by Tomasz Kmiec).
Susceptibility-weighted MRI demonstrates involvement of nigropallidal pathways [29]. Multiple system atrophy and cortical basal ganglionic degeneration may exhibit similar lesion, but clearly differ in clinical course or other imaging features $[30,31]$.

\section{TREATMENT}

Until now, there is no specific treatment for PKAN. Oral or intrathecal baclofen, stereotactic pallidotomy and anticholinergic treatment may provide symptomatic relief but have no known disease-modifying effects $[32,33]$.

Iron chelators which cross the blood brain barrier are an obvious option for pathophysiologically founded treatment. Treatment with deferiprone led to radiological and clinical improvement in two idiopathic NBIA patients [34, 35]. An unblinded, single-arm pilot study of deferiprone in 10 PKAN patients showed good tolerability and significant median reduction of globus pallidus iron of $30 \%(\mathrm{p}=0.008)$ after 6 months treatment, but no change in clinical scores [36]. Another unblinded, single-arm pilot study in NBIA including four PKAN cases underlined the safety and tolerability of deferiprone, and revealed mild-to-moderate clinical improvement in 2 of the 4 PKAN patients [37]. These results warrant a large, adequately powered, randomized, doubleblind trial which will start in 2012. The trial is conducted by an international consortium (TIRCON: Treat Iron-Related Childhood-Onset Neurodegeneration) including the authors and funded by the European Union.

While iron chelation represents the most feasible and promising candidate therapy for NBIA today, it remains unclear to what extent iron removal affects the phenotype. A treatment in PKAN that targets the primary defect may be pantethine which acts as an alternative precursor for CoA synthesis bypassing $P A N K 2$, the defective enzyme in PKAN. In $E$. coli a pantothenate kinase phosphoralates both pantothenate and pantethine [38]. In a Drosophila model pantethine treatment led to rescue of neurodegeneration and increase in life span which argues for an alternative kinase or pathway for pantothenate metabolism in the fly and possibly in human [39].

Finally, in view of the oxidative stress in NBIA that is presumably triggered by iron via the Fenton reaction, treatment with antioxidants may be an option but this has not been evaluated so far neither in vitro nor in vivo.

\section{PANTOTHENATE KINASE AND COA BIOSYNTHE- SIS}

The phosphorylation of pantothenate (Vitamine B5) represents the first and crucial (rate-limiting) step of CoA biosynthesis (Fig. 2). The other four steps which all take place in the cytosole are catalyzed by PPCS, PPCDC and the bifunctional enzyme PPAT-DPCK. CoA is a crucial coenzyme of a large number of oxidative and synthetic enzymes, in pathways like the citric acid cycle, sterol biosynthesis, amino acid metabolism, fatty acid biosynthesis and oxidation.

The human genome contains four genes which encode active pantothenate phosphorylating enzymes. These enzymes belonging to the pantothenate kinase family contain a highly conserved catalytic domain (approx. 83\% identical) 
but differ in the N-terminus which probably serves regulating purposes [40, 41]. PANK1 and PANK3 are cytoplasmatic enzymes. A forth related enzyme (PANK4) misses one Glu/Asp in its catalytic domain, in addition it harbours a carboxy-terminal domain with unknown function (COG1578 superfamily of unknown function, aa 450-733) [5]. Although in vitro data suggested an absence of kinase activity, the successful complementation of Drosophila and E. coli mutants confirmed its PANK activity [5, 42].

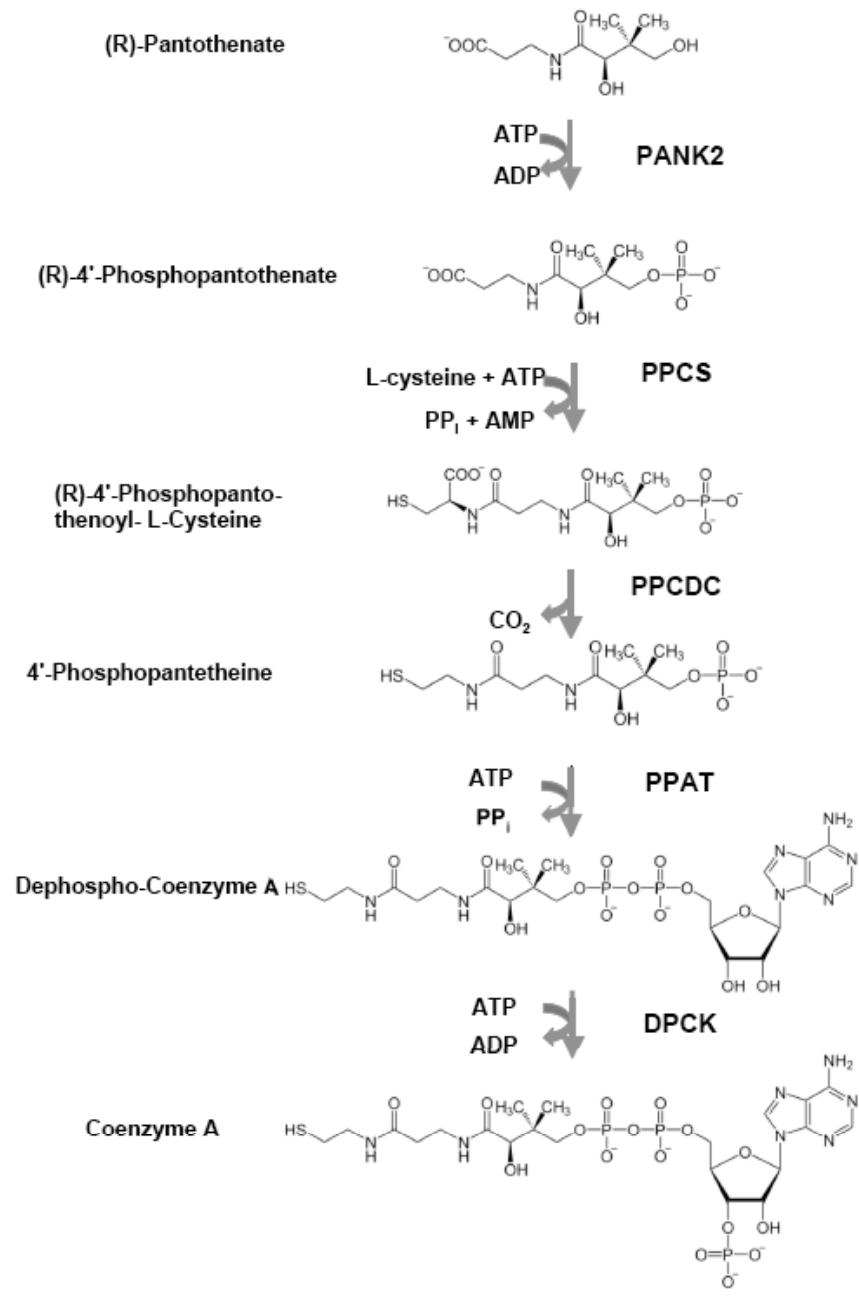

Fig. (2). CoA biosynthesis route.

For PANK1alpha and PANK3 the crystal structure is available [41]. Both pantothenate kinases are part of the actin fold protein kinase superfamily. They consist of two domains (A and B), the B-domain contains a dimerization interphase conferring a homodimer-configuration and an acetyl-CoA-binding-motif. Via acetyl-CoA-binding, ATPbinding is inhibited [43]. Thereby the activity of the pantothenate kinases is regulated by intracellular CoA levels which create a feedback regulation mechanism. On the other side, the presence of palmitoylcarnitine inhibits acetyl-CoA inhibition, thereby activating PANK2 [43].

The $63 \mathrm{kDa}$ precursor of $P A N K 2$ comprises in addition to the highly conserved catalytical domain a mitochondrial targeting signal $[5,44]$. Via a $59 \mathrm{kDa}$ intermediate it is processed in two steps in the mitochondrial matrix by the mitochondrial processing peptidases (MPP) to a final $48 \mathrm{kDa}$
$P A N K 2$, suggesting an intramitochondrial localization, in the intermembrane space or in the matrix. One argument in favour of an IMS localization is the fact that other proteins that also have two MPP binding sites are found in the IMS [43], together with the cytoplasmic localization of the other enzymes involved in the CoA biosynthesis. Via porin, an abundant membrane protein, 4'phosphopantothenate might be transported into the cytosole in order to serve the downstream pathway.

A shorter $P A N K 2$ isoform (288 aa) formed by an alternative exon 1 (1a) does not have a mitochondrial targeting signal [5]. Since no disease-causing mutation in exon 1a has been identified so far, the relevance of this isoform remains unclear.

Complementation experiments and in vitro enzymatic assays confirm kinase activity of PANK2 [3, 44]. Increased pantothenate serum levels have been measured in PKAN patients [45]. This finding argues in favour of pantothenate being the physiological substrate. Due to its ability to sense acetyl-CoA and palmitoylcarnitine levels, $P A N K 2$ might also function as a sensor of the mitochondrial demand for CoA [43].

\section{GENETICS}

Autosomal recessive mutations in $P A N K 2$ have been implicated with PKAN appearance. PANK2 spans $35 \mathrm{kB}$ of genomic DNA and contains 8 exons with alternate usage of exon $1 \mathrm{a}$ and $1 \mathrm{~b}$ [5]. According to the human gene mutation database in Cardiff (http://www.hgmd.org/) more than 100 mutations, including small deletions, duplications, missense mutations and splice site mutations have been identified. Hayflick et al. reported that about $10 \%$ of patients carry only one mutation and discussed the potential role of promoter mutations $[21,46]$. In about $5 \%(3 / 34)$ of cases exonic deletions were detected in European samples and so far no cases with only a single mutated allele [3]. Two different nomenclatures for mutations have been used in the literature based on different predicted translation initiation codons. We use the longer transcript (NM_153638) preceding the other one by 110 amino acids ( 330 nucleotides). The mutations are evenly distributed throughout the conserved parts of the $P A N K 2$ gene $[2,3]$. Most of the known mutations are private mutations. Hayflick et al. reported that two common mutations (c.1561G $>$ A, p.G521R and c.1583G $>$ A, p.T528M) account for 33\% (41/123) of all PKAN alleles [2]. A 1-bpdeletion (c.573delC, p.S191RfsX13) was frequently identified in patients from Poland which might be due to a founder effect [3]. Presence of null-mutations on both alleles strongly correlates with an early onset (younger than six years). In vitro cell culture assays have been performed to test $P A N K 2$ activity of different $P A N K 2$ alleles with the purpose of correlating disease severity to the level of pantothenate kinase activity. Some mutations like c.1561G $>$ A, p.G521R lead to a loss of kinase activity. Crystallographic mapping by Hong et al. revealed that the c. $1561 \mathrm{G}>\mathrm{A}, \mathrm{p} . \mathrm{G} 521 \mathrm{R}$ mutation affects the ATP binding site [41]. Correspondingly, this mutation leads to early onset. Other mutations result in only slightly reduced PANK2 activity. The mutation c.1583G $>A$, p.T528M which is located on the surface of PANK2 shows no discernible change of enzyme activity [44], but has a 
moderate effect on $P A N K 2$ stability and slightly reduced $P A N K 2$ activity in an $E$. coli experiment [3, 41]. Such mutations are associated with late onset when homozygous or compound heterozygous.

\section{TRANSGENIC ANIMALS}

As in human, the murine genome encodes four pantothenate kinases. However, unlike human $P A N K 2$, mouse Pank2 localizes to cytosole [47]. The difference in the subcellular localization is also reflected in a different phenotype. Mice with homozygous null mutations in Pank2 develop weight loss, retinal degeneration and infertility, but no neurological symptoms such as dystonia and no accumulation of iron in the central nervous system [48]. When challenged with a vitamin B5 deficient diet, wild type mice develop azoospermia, a low-lying pelvis and slow steps [49].

Human $P A N K 2$ is ubiquitously expressed with a high rate of expression in brain tissue, especially cerebellum and caudate nucleus. Murine Pank2 on the other hand, has a low expression in brain and a very high testicular expression [47]. The attenuated phenotype in mice might be explained by the specific expression patterns of Pank2 in mice.

The fly genome contains only one pantothenate kinase gene (fumble, fbl), which codes for five isoforms. One of those five isoforms localizes to mitochondria. Drosophila flies with a hypomorph fbl-allele show reduced pantothenate kinase activity, sterility in both sexes, movement disorders and neurodegeneration. Deaths usually occur in pupae stage or after eclosion. Electron microscopy shows swollen mitochondria and destroyed cristae and membranes. Introduction of human PANK3 and PANK4 which are both cytosolic enzymes in the fumble fly failed to completely reverse the phenotype which points to the relevance of a mitochondrial localized pantothenate kinase activity [42].

Rana and colleagues suggested that pantethine might work as a substrate for a $P A N K 2$ independent CoA biosynthesis. Treatment with pantethine restored all phenotypes in the dPANK/fumble fly including CoA deficiencies, neurological dysfunction and mitochondrial discrepancies [39]. Based on this promising finding, the therapeutical effectiveness of pantheteine is currently tested in mice.

\section{PATHOLOGY}

Most of the neuropathologic literature on NBIA is from a pre-molecular era making it difficult to draw firm conclusions on PKAN pathology. However, typical cases with the diagnosis Hallervorden-Spatz syndrome are likely to correspond to PKAN. Early autopsy cases were characterized by rust-brown pigmentation in the globus pallidus and the reticular zone of the substantia nigra, with iron being the major component of this pigment [50]. While these areas contain approximately three times the normal amount of iron, a global increase in brain iron is not seen and systemic iron metabolism is normal. In PKAN, spheroid bodies (thought to represent swollen axons) have been observed in the pallidonigral system as well as in the white and gray matter of the cerebrum [51]. Spheroid bodies in the peripheral nervous system, in contrast, point to the diagnosis of infantile neuroaxonal dystrophy (INAD, previously Seitelberger's dis- ease) which is due to mutations in the phospholipase A2, group VI (PLA2G6) gene. In a recent study, Kruer et al. described neuropathological findings in six cases of molecularly confirmed PKAN $[44,52]$. In contrast to INAD pathology, the PKAN pathology was restricted to the globus pallidus. Apart from iron and gliosis in the globus pallidus which corresponds to the radiological "eye of the tiger" sign, several distinctive features of PKAN pathology were found. Notably, Lewy bodies and other alpha-synuclein abnormalities were not encountered arguing against earlier perceptions of PKAN as a synucleinopathy. Similarly, neurofibrillary tangles and tau-positive neurites were also absent. In contrast, both tau-positive neuritis and Lewy bodies have been described in patients with C19orf12 mutations [12]. Ironladen macrophages were prominent in all PKAN cases, which may indicate an attempt to remove iron from the brain parenchyma. Intriguingly, increased amyloid precursor protein staining was found both in neuroaxonal spheroids and in neuronal processes. This finding may correspond to the recent recognition that amyloid precursor protein has a role in cellular iron efflux [53]. In addition, degenerating neurons were strongly positive for ubiquitin which points to accumulation of another abnormal and yet unassigned protein.

Obviously, the pathophysiological mechanisms underlying iron deposition in PKAN are of great interest considering the iron accumulation in more common neurodegenerative disorders, such as Alzheimer and Parkinson disease (PD).

While in PKAN the iron accumulation is located in globus pallidus, in PD the iron accumulation is detected in the substantia nigra. The pathological relevance of iron accumulation is supported by (i) the localization: iron changes are seen in the pars compacta of the substantia nigra which is the main site of pathogenesis in PD harbouring pigmented neurons which are involved in PD pathogenesis [54]. In comparison, age-related iron accumulation in normal controls occurs mainly in the pars reticulata in the substantia nigra [55-57]. Second, the correlation of iron accumulation with disease severity and motor score in the contralateral body side underlines its pathological relevance [58, 59]. However, disease duration is not correlated with iron accumulation in PD patients [60]. Interestingly, in patients with Alzheimer Disease, the development of parkinsonism is associated with accumulation of iron in the substantia nigra [61]. In principal, the hippocampus is the primarily affected site in Alzheimer Diseases [55, 62].

No nigral iron accumulaton has been identified in presymptomatic PD or mild PD indicating that iron accumulation in PD may be a secondary phenomenon $[63,64]$. On the contrary, in PKAN MRI changes have been found in presymptomatic individuals [28].

\section{PATHOGENESIS}

Neuroferritinopathy and aceruloplasminemia can be attributed directly to an impairment of proteins which are involved in iron metabolism $[10,11,16,18]$. For the other NBIA disorders, the link from gene defect to iron accumulation is less clear.

Several hypotheses regarding the genesis of PKAN pathology have been discussed. The first concentrates on the 
loss of the key enzyme of the biosynthesis of CoA. The resulting cellular CoA deficiency, which can be reproduced in the Drosophila and mammalian cell culture [39,65] may hamper beta-oxidation, thereby cellular energy and lipid metabolism since CoA serves as a key substrate for many cellular reactions.

Since the identification of further NBIA genes in the last three years, the role of lipid metabolism has received increasing attention in the pathogenesis of NBIA (Fig. 3). In addition, the mitochondria have taken centre stage following the verification of the mitochondrial localization of $P A N K 2$, PLA2G6 and the recently identified C19orf12 [5, 12, 66]. Although defects of the mitochondrial respiratory chain have not been identified in PKAN patients, elevation of lactate in plasma of a PKAN cohort compared to a control group point to mitochondrial dysfunction in PKAN patients [45]. In addition, defects in bile acid conjugation and lipid metabolism were shown in global metabolic profiling in fibroblasts of PKAN patients [45].

Insufficient remodeling and degeneration of mitochondrial inner membranes and presynaptic membranes has been shown in iPLA(2) $\beta$ knock-out (KO) mice [67] and mitochondrial structure and function are impaired in $\mathrm{dPANK} / \mathrm{fbl}$ mutants [39].

The PLA2G6 gene encodes a calcium independent phospholipase [14] and leads to PLA2G6- associated neurodegeneration $[14,17]$. PLA2G6 is a crucial enzyme in membrane homeostasis, for instance by remodelling of membrane phospholipids [68]. Involvement of PLA2G6 in cardiolipin metabolism has been shown in Drosophila. In eukaryotes, cardiolipin is exclusively found in the mitochondrial membrane and crucial for proper mitochondrial function [69]. Taffazin- deficient-Drosophila, a model of Barth syndrome, exhibit low cardiolipin concentration, abnormal cardiolipin fatty acyl composition, abnormal mitochondria, and poor motor function [70-72]. This phenotype of the taffazin knockout is rescued by an additional inactivation of the PLA2G6 gene.

Bras et al. suggested that PANK2, PLA2G6 and Parkinson's Disease (PD) genes might be partners in a complex network of ceramide metabolism (Fig. 3). Ceramide metabolism has been linked to the pathogenesis of PD ever since heterozygous mutations in glucocerebrosidase (GBA) have been identified as a risk factor for PD [73, 74]. Galactosylceramide and sulfatide are major components of myelin and play a critical role in its maintainance $[75,76]$. Key substrate for generation of ceramide is CoA which establishes the link of ceramide metabolism to PKAN [77]. Involvement of PLA2G6 in the ceramide pathway has been implicated since PLA2G6 promotes ceramide generation via neutral sphingomyelinase-catalyzed hydrolysis of sphingomyelins [78].

Recently three further NBIA genes have been identified which can be linked to lipid and ceramide metabolism. $\mathrm{Mu}-$ tations in the $F A 2 H$ gene have been identified in one subtype of NBIA named fatty acid hydroxylase-associated neurodegeneration (FAHN) [13]. FA2H encodes fatty acid 2hydroxylase which is part of the ceramide biosynthesis due to its function to generate 2-hydroxylated fatty acids for integration in 2-hydroxydihydroceramide and 2-hydroxyceramide.

The ATP13A2 gene is mutated in patients with KuforRakeb syndrome which has recently been identified to be part of the NBIA spectrum [15]. ATP13A2 mutations have been shown to cause a late onset neurodegenerative phenotype in dogs [79]. ATP13A2 encodes a lysosomal divalent cation transporter [80]. Kruer et al. discussed that mutations in ATP13A2 might lead to impaired membrane lipid recycling, and impaired autophagy and accentuated a-synuclein toxicity [13]. A recent study showed decreased ATP synthesis rates, increased mitochondrial DNA levels, a higher fre-

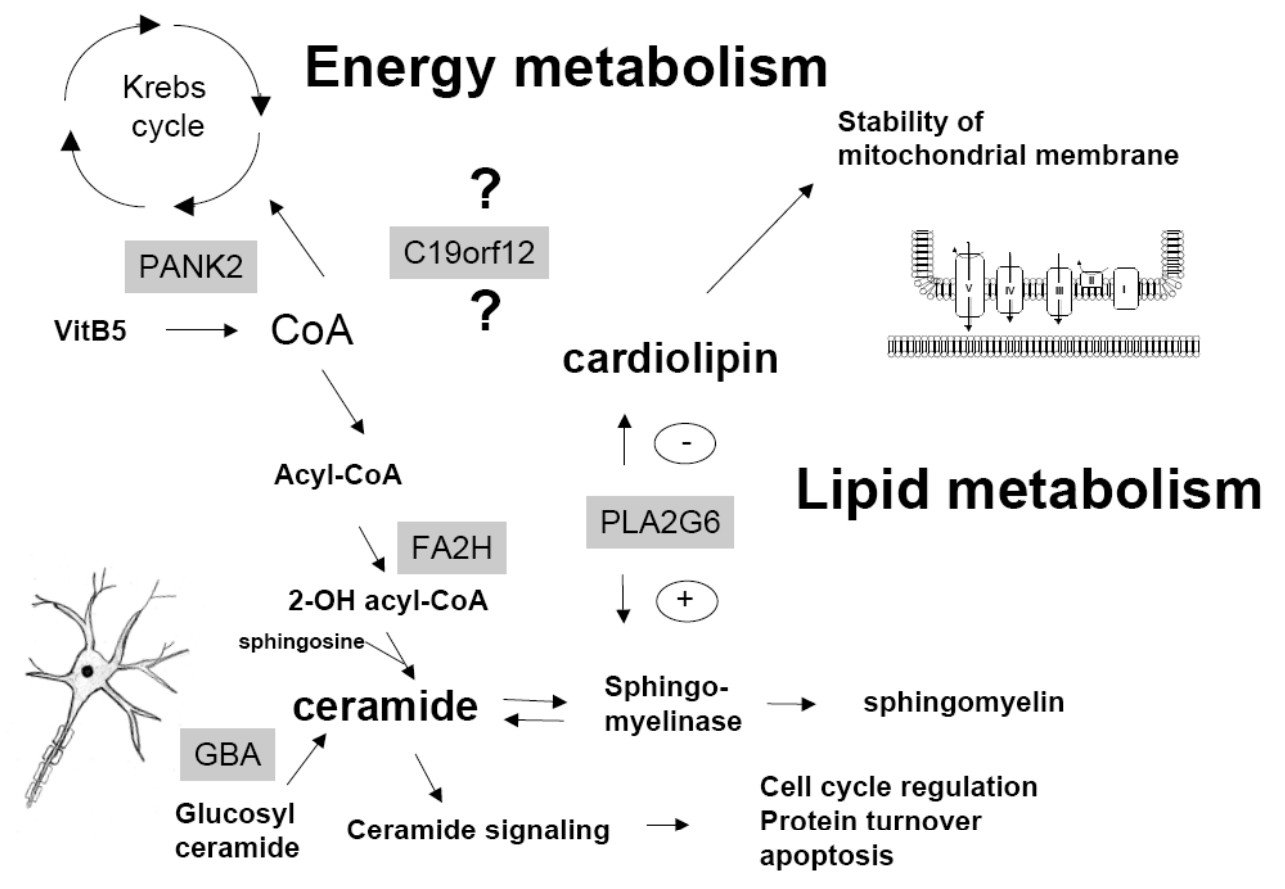

Fig. (3). Pathogenesis of NBIA: a schematic overview of interconnections of NBIA genes (adapted from Kruer et al. 2011). 
quency of mitochondrial DNA lesions, increased oxygen consumption rates, and increased fragmentation of the mitochondrial network in fibroblasts from patients with ATP13A2 mutations [81].

Mutations in C19orf12 are the second frequent cause for NBIA after PANK2. The protein encoded by this orphan gene is localised to mitochondria and expression analysis in blood points to mitochondrial processes related to $\mathrm{CoA}$ [12].

As shown in (Fig. 3) the identification of further NBIA genes suggest impaired mitochondrial lipid metabolism as an important pathophysiological mechanism. Under certain circumstances impaired mitochondrial lipid metabolism is able to affect mitochondrial energy metabolism in a tissue specific manner as shown for the cardiolipin methyltransferase taffazin and the acylglycerolkinase (AGK) $[69,82]$.

Another hypothesis concentrates on one of the most prominent gaps in NBIA research: the iron accumulation. It is still unknown whether iron accumulation is a primary trigger of PKAN pathogenesis or rather a pathogenic end product. Physiologically, there is an age related increase in iron content in the brain [56], primarily in the globus pallidus, red nucleus, substantia nigra pars reticulate, dentate nucleus, and putamen. In the developing brain, iron is especially high in oligodendrocytes and is probably needed for the high energy demands of myelin synthesis [83]. Restriction of iron in diet leads to hypomyelination in rat models [84, 85]. Via iron accumulation in macrophages iron is delivered to maturating oligodendroglial progrenitors in the process of oligodendrogenesis [86]. Iron accumulation in NBIA might be a consequence of disturbance of lipid metabolism and consequently myelin breakdown and increased myelin turnover [87].

In the second step of the CoA pathway cysteine is incorporated into 4 phosphopantothenic acid. It was discussed that deficiency of $P A N K 2$ activity may lead to accumulation of cysteine and subsequently iron due to chelation $[1,88]$. Autooxidation in the presence of iron leads to the generation of free radicals. Involvement of oxidative stress in PKAN pathogenesis has been shown in the Drosophila. dPPCS and dPANK/fbl-Drosophila-mutants but not PPAT-DPCKmutants are hypersensitive to cysteine. Additionally, overexpression of ROS scavengers resulted in a protective effect against cysteine induced lethality in this model [89].

One of the remaining questions is why an alteration of a ubiquitously expressed gene like $P A N K 2$ results in an isolated brain disorder. The neurological phenotype of PKAN might be explained by (i) the pronounced sensitivity of the brain in particular the basal ganglia to oxidative stress added by the high energy demand and by the physiological high iron content of this organ [90] and by (ii) the specific high $P A N K 2$ expression levels in brain which accounts for $45 \%$ of PANK activity.

\section{CONCLUSION}

Though our understanding of etiology and phenotype of PKAN has greatly improved, the precise pathomechanisms leading from the gene defect to the clinical manifestations are still a matter of debate. The challenge in the future will be to unravel the exact function of $P A N K 2$ and its interaction with the other NBIA genes. A better understanding of these processes may open novel avenues for further therapeutic research in NBIA patients. As we gain more understanding of NBIA pathogenesis, we might advance our knowledge about more common neurodegenerative disorders like Parkinsons and Alzheimers Disease.

\section{ACKNOWLEDGMENT}

Financial support by the European Commission (FP7Health-F2-2011, project TIRCON, Grant Agreement no. 277984 ) is gratefully acknowledged.

\section{CONFLICT OF INTEREST}

None declared.
ABBREVIATION
PKAN = Pantothenate kinase-associated neurodegenera- tion
NBIA = Neurodegeneration with brain iron accumulation
PPCS $=4^{\prime}$-phosphopantothenoylcysteine synthetase
PPCDC $=(\mathrm{R})-4^{\prime}$-phospho-N-pantothenoylcysteine decar- boxylase
PPAT $=4^{\prime}$-phosphopantetheine adenylyltransferase
DPCK = Dephospho-CoA kinase
$\mathrm{PD}=$ Parkinson Disease

\section{REFERENCES}

[1] Zhou B, Westaway SK, Levinson B, et al. A novel pantothenate kinase gene $(P A N K 2)$ is defective in Hallervorden-Spatz syndrome. Nat Genet 2001; 28(4): 345-9.

[2] Hayflick SJ, Westaway SK, Levinson B, et al. Genetic, clinical, and radiographic delineation of Hallervorden-Spatz syndrome. $\mathrm{N}$ Engl J Med 2003; 348(1): 33-40.

[3] Hartig MB, Hortnagel K, Garavaglia B, et al. Genotypic and phenotypic spectrum of PANK2 mutations in patients with neurodegeneration with brain iron accumulation. Ann Neurol 2006; 59(2): 248-56.

[4] Gregory A, Hayflick SJ. Pantothenate Kinase-Associated Neurodegeneration In: GeneReviews at GeneTests: Medical Genetics Information Resource (database online) Copyright, University of Washington, Seattle 1997-2011 Available at http: //wwwgenetestsorg Updated March 23, 2010.

[5] Hortnagel K, Prokisch $\mathrm{H}$, Meitinger $\mathrm{T}$. An isoform of $\mathrm{h} P A N K 2$, deficient in pantothenate kinase-associated neurodegeneration, localizes to mitochondria. Hum Mol Genet 2003; 12(3): 321-7.

[6] Sian-Hulsmann J, Mandel S, Youdim MB, Riederer P. The relevance of iron in the pathogenesis of Parkinson's disease. J Neurochem 2011; 118(6): 939-57.

[7] Bush AI. The metallobiology of Alzheimer's disease. Trends Neurosci $2003 ; 26(4)$ : 207-14.

[8] Liu B, Moloney A, Meehan S, et al. Iron promotes the toxicity of amyloid beta peptide by impeding its ordered aggregation. J Biol Chem 2011; 286(6): 4248-56.

[9] Alazami AM, Al-Saif A, Al-Semari A, et al. Mutations in C2orf37, encoding a nucleolar protein, cause hypogonadism, alopecia, diabetes mellitus, mental retardation, and extrapyramidal syndrome. Am J Hum Genet 2008; 83(6): 684-91.

[10] Curtis AR, Fey C, Morris CM, et al. Mutation in the gene encoding ferritin light polypeptide causes dominant adult-onset basal ganglia disease. Nat Genet 2001; 28(4): 350-4.

[11] Harris ZL, Takahashi Y, Miyajima H, et al. Aceruloplasminemia: molecular characterization of this disorder of iron metabolism. Proc Natl Acad Sci U S A 1995; 92(7): 2539-43. 
[12] Hartig MB, Iuso A, Haack T, et al. Absence of an orphan mitochondrial protein, c19orf12, causes a distinct clinical subtype of neurodegeneration with brain iron accumulation. Am J Hum Genet 2011; 89(4): 543-50.

[13] Kruer MC, Paisan-Ruiz C, Boddaert N, et al. Defective FA2H leads to a novel form of neurodegeneration with brain iron accumulation (NBIA). Ann Neurol 2010; 68(5): 611-8.

[14] Morgan NV, Westaway SK, Morton JE, et al. PLA2G6, encoding a phospholipase $\mathrm{A} 2$, is mutated in neurodegenerative disorders with high brain iron. Nat Genet 2006; 38(7): 752-4.

[15] Schneider SA, Paisan-Ruiz C, Quinn NP, et al. ATP13A2 mutations (PARK9) cause neurodegeneration with brain iron accumulation. Mov Disord 2010; 25(8): 979-84.

[16] Kono S. Aceruloplasminemia. Current Drug Targets; 2012; 13(7): ???-???.

[17] McNeill A. PLA2G6 mutations and other rare causes of Neurodegeneration with Brain Iron Accumulation. Current Drug Targets; 2012; 13(7): ???-???.

[18] McNeill A, Chinnery P. Neuroferritinopathy. Current Drug Targets; $\operatorname{xxx}(\mathrm{xxx})$.

[19] Kauther KM, Hoft C, Rissling I, Oertel WH, Moller JC. The PLA2G6 gene in early-onset Parkinson's disease. Mov Disord 2011.

[20] Freeman K, Gregory A, Turner A, et al. Intellectual and adaptive behaviour functioning in pantothenate kinase-associated neurodegeneration. J Intellect Disabil Res 2007; 51(Pt. 6): 417-26.

[21] Gregory A, Polster BJ, Hayflick SJ. Clinical and genetic delineation of neurodegeneration with brain iron accumulation. J Med Genet 2009; 46(2): 73-80.

[22] Pellecchia MT, Valente EM, Cif L, et al. The diverse phenotype and genotype of pantothenate kinase-associated neurodegeneration. Neurology 2005; 64(10): 1810-2.

[23] Houlden H, Lincoln S, Farrer M, et al. Compound heterozygous PANK2 mutations confirm HARP and Hallervorden-Spatz syndromes are allelic. Neurology 2003; 61(10): 1423-6.

[24] Schipper HM. Neurodegeneration with brain iron accumulation Clinical syndromes and neuroimaging. Biochim Biophys Acta 2012; 1822(3): 350-60.

[25] McNeill A, Birchall D, Hayflick SJ, et al. T2* and FSE MRI distinguishes four subtypes of neurodegeneration with brain iron accumulation. Neurology 2008; 70(18): 1614-9.

[26] Hayflick SJ. Unraveling the Hallervorden-Spatz syndrome: pantothenate kinase-associated neurodegeneration is the name. Curr Opin Pediatr 2003; 15(6): 572-7.

[27] Baumeister FA, Auer DP, Hortnagel K, Freisinger P, Meitinger T. The eye-of-the-tiger sign is not a reliable disease marker for Hallervorden-Spatz syndrome. Neuropediatrics 2005; 36(3): 221-2.

[28] Hayflick SJ, Penzien JM, Michl W, et al. Cranial MRI changes may precede symptoms in Hallervorden-Spatz syndrome. Pediatr Neurol 2001; 25(2): 166-9.

[29] Vinod Desai S, Bindu PS, Ravishankar S, Jayakumar PN, Pal PK. Relaxation and susceptibility MRI characteristics in HallervordenSpatz syndrome. J Magn Reson Imaging 2007; 25(4): 715-20.

[30] Strecker K, Hesse S, Wegner F, et al. Eye of the tiger sign in multiple system atrophy. Eur J Neurol 2007; 14(11): e1-2.

[31] Molinuevo JL, Munoz E, Valldeoriola F, Tolosa E. The eye of the tiger sign in cortical-basal ganglionic degeneration. Mov Disord 1999; 14(1): 169-71.

[32] Timmermann L, Pauls KA, Wieland K, et al. Dystonia in neurodegeneration with brain iron accumulation: outcome of bilateral pallidal stimulation. Brain 2010; 133(Pt 3): 701-12.

[33] Lyoo $\mathrm{CH}$, Prokisch $\mathrm{H}$, Meitinger $\mathrm{T}$, et al. Anticholinergicresponsive gait freezing in a patient with pantothenate kinaseassociated neurodegeneration. Mov Disord 2008; 23(2): 283-4.

[34] Forni GL, Balocco M, Cremonesi L, et al. Regression of symptoms after selective iron chelation therapy in a case of neurodegeneration with brain iron accumulation. Mov Disord 2008; 23(6): 904-7.

[35] Kwiatkowski JL. Real-world use of iron chelators. Hematology Am Soc Hematol Educ Program; 2011: 451-8

[36] Zorzi G, Zibordi F, Chiapparini L, et al. Iron-related MRI images in patients with pantothenate kinase-associated neurodegeneration (PKAN) treated with deferiprone: Results of a phase II pilot trial. Mov Disord 2011; 26(9): 1755-9.
[37] Abbruzzese G, Cossu G, Balocco M, et al. A pilot trial of deferiprone for neurodegeneration with brain iron accumulation. Haematologica 2011; 96(11): 1708-11.

[38] Balibar CJ, Hollis-Symynkywicz MF, Tao J. Pantethine rescues phosphopantothenoylcysteine synthetase and phosphopantothenoylcysteine decarboxylase deficiency in Escherichia coli but not in Pseudomonas aeruginosa. J Bacteriol 2011; 193(13): 3304-12.

[39] Rana A, Seinen E, Siudeja K, et al. Pantethine rescues a Drosophila model for pantothenate kinase-associated neurodegeneration. Proc Natl Acad Sci U S A 2010; 107(15): 6988-93.

[40] Rock CO, Karim MA, Zhang YM, Jackowski S. The murine pantothenate kinase (Pank1) gene encodes two differentially regulated pantothenate kinase isozymes. Gene 2002; 291(1-2): 35-43.

[41] Hong BS, Senisterra G, Rabeh WM, et al. Crystal structures of human pantothenate kinases. Insights into allosteric regulation and mutations linked to a neurodegeneration disorder. J Biol Chem 2007; 282(38): 27984-93.

[42] Wu Z, Li C, Lv S, Zhou B. Pantothenate kinase-associated neurodegeneration: insights from a Drosophila model. Hum Mol Genet 2009; 18(19): 3659-72.

[43] Leonardi R, Rock CO, Jackowski S, Zhang YM. Activation of human mitochondrial pantothenate kinase 2 by palmitoylcarnitine. Proc Natl Acad Sci U S A 2007; 104(5): 1494-9.

[44] Kotzbauer PT, Truax AC, Trojanowski JQ, Lee VM. Altered neuronal mitochondrial coenzyme A synthesis in neurodegeneration with brain iron accumulation caused by abnormal processing, stability, and catalytic activity of mutant pantothenate kinase 2 . J Neurosci $2005 ; 25(3)$ : 689-98.

[45] Leoni V, Strittmatter L, Zorzi G, et al. Metabolic consequences of mitochondrial coenzyme A deficiency in patients with PANK2 mutations. Mol Genet Metab 2012.

[46] Polster BJ, Yoon MY, Hayflick SJ. Characterization of the human $P A N K 2$ promoter. Gene 2010; 465(1-2): 53-60.

[47] Leonardi R, Zhang YM, Lykidis A, Rock CO, Jackowski S. Localization and regulation of mouse pantothenate kinase 2. FEBS Lett 2007; 581(24): 4639-44.

[48] Kuo YM, Duncan JL, Westaway SK, et al. Deficiency of pantothenate kinase 2 (Pank2) in mice leads to retinal degeneration and azoospermia. Hum Mol Genet 2005; 14(1): 49-57.

[49] Kuo YM, Hayflick SJ, Gitschier J. Deprivation of pantothenic acid elicits a movement disorder and azoospermia in a mouse model of pantothenate kinase-associated neurodegeneration. J Inherit Metab Dis 2007; 30(3): 310-7.

[50] Hallervorden J. Uber eine familiare Erkrankung im extrapyramidalen System. Dtsch Z Nervenheilkd 1924; 81: 204-10.

[51] Swaiman KF. Hallervorden-Spatz syndrome. Pediatr Neurol 2001; 25(2): 102-8

[52] Kruer MC, Hiken M, Gregory A, et al. Novel histopathologic findings in molecularly-confirmed pantothenate kinase-associated neurodegeneration. Brain 2011; 134(Pt 4): 947-58.

[53] Duce JA, Bush AI. Biological metals and Alzheimer's disease: implications for therapeutics and diagnostics. Prog Neurobiol 2010; 92(1): 1-18.

[54] Dexter DT, Carayon A, Javoy-Agid F, et al. Alterations in the levels of iron, ferritin and other trace metals in Parkinson's disease and other neurodegenerative diseases affecting the basal ganglia. Brain 1991; 114 ( Pt 4): 1953-75.

[55] Schenck JF, Zimmerman EA. High-field magnetic resonance imaging of brain iron: birth of a biomarker? NMR Biomed 2004; 17(7): 433-45.

[56] Hallgren B, Sourander P. The effect of age on the non-haemin iron in the human brain. J Neurochem 1958; 3(1): 41-51.

[57] Wang H, Qian WJ, Chin MH, et al. Characterization of the mouse brain proteome using global proteomic analysis complemented with cysteinyl-peptide enrichment. J Proteome Res 2006; 5(2): 361-9.

[58] Gorell JM, Ordidge RJ, Brown GG, et al. Increased iron-related MRI contrast in the substantia nigra in Parkinson's disease. Neurology $1995 ; 45(6)$ : 1138-43.

[59] Jin L, Wang J, Zhao L, et al. Decreased serum ceruloplasmin levels characteristically aggravate nigral iron deposition in Parkinson's disease. Brain 2011; 134(Pt 1): 50-8. 
[60] Wallis LI, Paley MN, Graham JM, et al. MRI assessment of basal ganglia iron deposition in Parkinson's disease. J Magn Reson Imaging 2008; 28(5): 1061-7.

[61] Brar S, Henderson D, Schenck J, Zimmerman EA. Iron accumulation in the substantia nigra of patients with Alzheimer disease and parkinsonism. Arch Neurol 2009; 66(3): 371-4.

[62] Bartzokis G. Age-related myelin breakdown: a developmental model of cognitive decline and Alzheimer's disease. Neurobiol Aging 2004; 25(1): 5-18; author reply 49-62.

[63] Dexter DT, Jenner P, Schapira AH, Marsden CD. Alterations in levels of iron, ferritin, and other trace metals in neurodegenerative diseases affecting the basal ganglia. The Royal Kings and Queens Parkinson's Disease Research Group. Ann Neurol 1992; 32 Suppl: S94-100.

[64] Riederer P, Sofic E, Rausch WD, et al. Transition metals, ferritin, glutathione, and ascorbic acid in parkinsonian brains. J Neurochem 1989; 52(2): 515-20.

[65] Zhang YM, Chohnan S, Virga KG, et al. Chemical knockout of pantothenate kinase reveals the metabolic and genetic program responsible for hepatic coenzyme A homeostasis. Chem Biol 2007; 14(3): 291-302.

[66] Seleznev K, Zhao C, Zhang XH, Song K, Ma ZA. Calciumindependent phospholipase A2 localizes in and protects mitochondria during apoptotic induction by staurosporine. J Biol Chem 2006; 281(31): 22275-88.

[67] Beck G, Sugiura Y, Shinzawa K, et al. Neuroaxonal Dystrophy in Calcium-Independent Phospholipase A2 \{beta\} Deficiency Results from Insufficient Remodeling and Degeneration of Mitochondrial and Presynaptic Membranes. J Neurosci; 31(31): 11411-20.

[68] Balsinde J, Balboa MA, Dennis EA. Antisense inhibition of group VI Ca2+-independent phospholipase A2 blocks phospholipid fatty acid remodeling in murine P388D1 macrophages. J Biol Chem 1997; 272(46): 29317-21.

[69] Houtkooper RH, Vaz FM. Cardiolipin, the heart of mitochondrial metabolism. Cell Mol Life Sci 2008; 65(16): 2493-506.

[70] Bione S, D'Adamo P, Maestrini E, et al. A novel X-linked gene, G4.5. is responsible for Barth syndrome. Nat Genet 1996; 12(4): 385-9.

[71] Malhotra A, Edelman-Novemsky I, Xu Y, et al. Role of calciumindependent phospholipase A2 in the pathogenesis of Barth syndrome. Proc Natl Acad Sci U S A 2009; 106(7): 2337-41.

[72] Xu Y, Condell M, Plesken H, et al. A Drosophila model of Barth syndrome. Proc Natl Acad Sci U S A 2006; 103(31): 11584-8.

[73] Lwin A, Orvisky E, Goker-Alpan O, LaMarca ME, Sidransky E. Glucocerebrosidase mutations in subjects with parkinsonism. Mol Genet Metab 2004; 81(1): 70-3

[74] Bras J, Singleton A, Cookson MR, Hardy J. Emerging pathways in genetic Parkinson's disease: Potential role of ceramide metabolism in Lewy body disease. FEBS J 2008; 275(23): 5767-73.

[75] Coetzee T, Fujita N, Dupree J, et al. Myelination in the absence of galactocerebroside and sulfatide: normal structure with abnormal function and regional instability. Cell 1996; 86(2): 209-19.
[76] Bosio A, Binczek E, Stoffel W. Functional breakdown of the lipid bilayer of the myelin membrane in central and peripheral nervous system by disrupted galactocerebroside synthesis. Proc Natl Acad Sci U S A 1996; 93(23): 13280-5.

[77] Hannun YA, Obeid LM. Principles of bioactive lipid signalling: lessons from sphingolipids. Nat Rev Mol Cell Biol 2008; 9(2): 13950 .

[78] Lei X, Zhang S, Bohrer A, et al. The group VIA calciumindependent phospholipase A2 participates in ER stress-induced INS-1 insulinoma cell apoptosis by promoting ceramide generation via hydrolysis of sphingomyelins by neutral sphingomyelinase. Biochemistry 2007; 46(35): 10170-85.

[79] Wohlke A, Philipp U, Bock P, et al. A one base pair deletion in the canine ATP13A2 gene causes exon skipping and late-onset neuronal ceroid lipofuscinosis in the Tibetan terrier. PLoS Genet 2011; 7(10): e1002304.

[80] Schmidt K, Wolfe DM, Stiller B, Pearce DA. Cd2+, Mn2+, Ni2+ and Se2+ toxicity to Saccharomyces cerevisiae lacking YPK9p the orthologue of human ATP13A2. Biochem Biophys Res Commun 2009; 383(2): 198-202.

[81] Grunewald A, Arns B, Seibler P, et al. ATP13A2 mutations impair mitochondrial function in fibroblasts from patients with KuforRakeb syndrome. Neurobiol Aging 2012.

[82] Mayr JA, Haack TB, Graf E, et al. Lack of the Mitochondrial Protein Acylglycerol Kinase Causes Sengers Syndrome. Am J Hum Genet. 2012; 90(2): 314-20.

[83] Todorich B, Pasquini JM, Garcia CI, Paez PM, Connor JR. Oligodendrocytes and myelination: the role of iron. Glia 2009; 57(5): 467-78.

[84] Yu GS, Steinkirchner TM, Rao GA, Larkin EC. Effect of prenatal iron deficiency on myelination in rat pups. Am J Pathol 1986; 125(3): 620-4.

[85] Ortiz E, Pasquini JM, Thompson K, et al. Effect of manipulation of iron storage, transport, or availability on myelin composition and brain iron content in three different animal models. J Neurosci Res 2004; 77(5): 681-9.

[86] Schonberg DL, McTigue DM. Iron is essential for oligodendrocyte genesis following intraspinal macrophage activation. Exp Neurol 2009; 218(1): 64-74.

[87] Dusek P, Jankovic J, Le W. Iron dysregulation in movement disorders. Neurobiol Dis 2012.

[88] Johnson MA, Kuo YM, Westaway SK, et al. Mitochondrial localization of human PANK2 and hypotheses of secondary iron accumulation in pantothenate kinase-associated neurodegeneration. Ann N Y Acad Sci 2004; 1012: 282-98.

[89] Bosveld F, Rana A, van der Wouden PE, et al. De novo CoA biosynthesis is required to maintain DNA integrity during development of the Drosophila nervous system. Hum Mol Genet 2008; 17(13): 2058-69.

[90] Rouault TA, Cooperman S. Brain iron metabolism. Semin Pediatr Neurol 2006; 13(3): 142-8. 